15

\title{
Особенности подготовки камер хранения ультрахолодных нейтронов и чувствительность ЭДМ-спектрометра
}

\author{
(C) М.С. Ласаков, А.Н. Пирожков, А.П. Серебров \\ Национальный исследовательский центр „Курчатовский институт“ - Петербургский институт ядерной фризики \\ им. Б.П. Константинова, \\ 188300 Гатчина, Ленинградская обл., Россия \\ ฯ e-mail: pirozhkov_an@pnpi.nrcki.ru
}

(Поступило в Редакцию 13 июля 2018 г.)

В эксперименте по поиску электрического дипольного момента (ЭДМ) нейтрона с использованием ультрахолодных нейтронов $(\mathrm{УХH})$ важнейшей частью установки являются ячейки хранения УХН. Изучалось влияние технологии подготовки и напыления элементов ячеек хранения УХН на предельное рабочее значение высокого напряжения и способность этих ячеек к долговременной работе в условиях высокого напряжения с коммутацией полярности. На рабочем варианте камеры хранения нейтронов ЭДМ-спектрометра получен результат $\pm 250 \mathrm{kV}$ при высоте ячейки $9 \mathrm{~cm}$.

DOI: 10.21883/JTF.2019.03.47188.283-18

Эксперимент по поиску электрического дипольного момента нейтрона является одним из важнейших в неускорительной физике элементарных частиц и дает ограничения на различные модели нарушения $C P$ и $T$-инвариантностей в теоретической физике. Обнаружение отличного от нуля электрического дипольного момента (ЭДМ) нейтрона может явиться решающим фактором в выборе вариантов расширения Стандартной модели, понимании свойств и природы фундаментальных сил, что важно также для адекватного описания эволюции Вселенной на ранних стадиях (барионная асимметрия Вселенной). Лучшее на сегодняшний день экспериментальное ограничение на величину ЭДМ-нейтрона было получено на высокопоточном реакторе института Лауэ-Ланжевена (ИЛЛ) в Гренобле на установке с хранением ультрахолодных нейтронов (УХН) международной коллаборацией RAL/Sussex/ILL: $\left|d_{n}\right|<2.9 \cdot 10^{-26} e \mathrm{~cm} \quad(90 \%$ C.L.) [1]. Измерения проводились при среднем значении величины напряженности электрического поля в рабочем объеме установки $10 \mathrm{kV} / \mathrm{cm}$ - важный фактор, существенно влияющий на чувствительность прибора.

Со схемой экспериментальной установки Петербургского института ядерной физики им. Б.П. Константинова (ПИЯФ) для измерения ЭДМ-нейтрона с использованием УХН и последними результатами, полученными на этом оборудовании, можно ознакомиться в работах $[2,3]$. Оставляя здесь без внимания необходимые, сложные и очень важные узлы экспериментальной установки, остановимся на главной ее части: симметричные относительно центрального электрода камеры хранения УХН, в которых и исследуется взаимодействие нейтронов с электрическим полем. Используется метод магнитного резонанса рамзеевского типа. Искомый экспериментальный эффект (ЭДМ-нейтрона) определяется по фазовому сдвигу резонансной кривой при реверсе направления электрического поля относительно постоянного магнит- ного поля, в котором и осуществляется магнитный резонанс. Малый сдвиг резонанса может быть зарегистрирован по изменению счета нейтронов в рабочей точке, которая выбирается на резонансной кривой на участке с максимальной производной.

Особенностью нашего спектрометра в отличие от [1] является наличие двух камер хранения УХН с общей системой магнитных полей и с равными по величине, но противоположно направленными электрическими полями. При изменении полярности электрического поля искомые эффекты от ЭДМ-нейтрона в разных камерах будут иметь противоположные знаки, в то время как нестабильность общих магнитных условий приводит к сдвигу резонансной частоты одного знака. Разность результатов измерений ЭДМ по отдельным камерам приводит к сложению эффектов от ЭДМ-нейтрона, при этом эффекты от коррелированного изменения счета, не связанного с ЭДМ, значительно подавляются, уменьшая итоговый разброс результатов измерений.

Статистическая погрешность эксперимента в общем случае определяется формулой

$$
\delta d_{n}=\frac{h}{2 \varepsilon \sqrt{N} E T},
$$

где $\varepsilon \leq 1$ - параметр, в общем случае характеризующий качество экспериментальной установки, он зависит от многих факторов (поляризация нейтронов, однородность и стабильность магнитного поля, фон, др.), $E$ - напряженность электрического поля, $T-$ время свободной прецессии (время хранения нейтронов в резонансных условиях - период резонансной кривой), $N$ - полный счет нейтронов за время эксперимента при обеих полярностях электрического поля.

Чем меньше статистическая ошибка, получаемая в процессе измерений в единицу времени (например, за сутки), тем выше чувствительность экспериментальной 
установки. Таким образом, чувствительность эксперимента пропорциональна времени хранения нейтронов в резонансе, напряженности электрического поля и имеет корневую зависимость от полного счета нейтронов. Отсюда следует, что вопрос о качестве покрытия рабочих поверхностей камер хранения УХН становится ключевым. С одной стороны, это должен быть материал, имеющий высокую граничную скорость отражения УХН, с другой - покрытие кольцевых изоляторов должно иметь высокую электрическую прочность и сопротивление.

История работ по поиску ЭДМ-нейтрона в ПИЯФ насчитывает несколько десятков лет с длительными перерывами, когда у нас не было источников ультрахолодных нейтронов и не было возможности проводить эксперимент вне ПИЯФ на других источниках УХН. Опыт работы с экспериментальной установкой приобретался непосредственно в процессе измерений и набора статистики, при этом специальных и сколько-нибудь длительных технологических исследований для решения конкретных проблем эксперимента ранее не проводилось. Ухудшение качества покрытия изоляторов и электродов в ходе экспериментов приводило к падению времени хранения нейтронов и уменьшению чувствительности установки. Это „лечилось“ жесткой очисткой рабочих поверхностей и их новым напылением, после чего вновь требовалась тренировка всей сборки. Но работа с коммутируемым высоким напряжением снова приводила к ухудшению качества напыления, а увеличение токов утечки и возникающие пробои заставляли снижать высокое напряжение. В итоге были большие потери полезного времени, а за длительный период измерений удавалось производить набор статистики при средних значениях напряженности электрического поля $12-14 \mathrm{kV} / \mathrm{cm}$.

В нашем ЭДМ спектрометре ячейки камер хранения образуются алюминиевыми электродами в виде дисков (диаметром $620 \mathrm{~mm}$ и толщиной $35 \mathrm{~mm}$ ) с закругленными краями и цилиндрическими кольцевыми изоляторами из плавленого кварца или ситалла (стеклокристаллический материал, „glassceramic“ [4]). Внутренний диаметр кольцевых изоляторов $526 \mathrm{~mm}$, толщина стенок 8-12 mm, высота колец около $100 \mathrm{~mm}$. На рисунке показана сборка камер хранения нейтронов и часть высоковольтного ввода в вакуумную камеру стенда высоковольтных испытаний [5]; представлен вариант сборки с нейтроноводами, которые размещаются в теле средних электродов.

Для фиксации изоляторов и уменьшения вероятности локальных перенапряжений и пробоев на границе „электрод-изолятор“ на поверхности электродов сделаны посадочные канавки глубиной $6 \mathrm{~mm}$. Мы использовали изоляторы, изготовленные из кварца и ситалла. По достигнутым результатам величины высокого напряжения и времени хранения нейтронов принципиальной разницы между этими материалами мы не обнаружили.

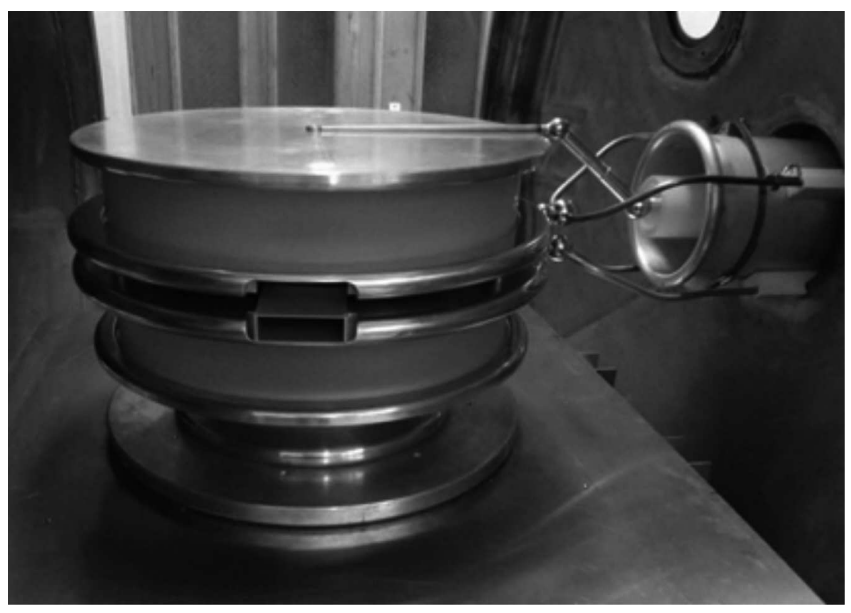

Сборка камер хранения УХН в вакуумной камере стенда для высоковольтных испытаний.

Однако изделия из кварца требуют более бережного обращения из-за своей хрупкости.

Для того чтобы обеспечить максимальную плотность и время удержания нейтронов в камерах хранения, полированная внутренняя поверхность алюминиевых электродов покрыта слоем бериллия, а поверхность изоляторов - слоем окиси бериллия. Эти материалы обеспечивают высокую граничную скорость отражения нейтронов $\left(v_{\text {critic }} \sim 6.8 \mathrm{~m} / \mathrm{s}\right)$.

К настоящему времени на созданном оборудовании проведены дополнительные исследования, которые позволили подобрать последовательность и режимы технологических процессов при подготовке камер хранения УХН для их работы в условиях максимальной напряженности электрического поля и с покрытиями, имеющими высокую граничную скорость отражения УХН. Для расширения возможностей испытательной установки каждый каскадный генератор высоковольтного источника [5] был дополнительно оборудован еще двумя ступенями умножения напряжения, что позволило увеличить выходное напряжение источника до $\pm 250 \mathrm{kV}$.

Мы рассмотрим три основные причины, существенно влияющие на чувствительность экспериментальной установки: микронеоднородность („звездчатость“) магнетронного напыления на изоляторы и электроды камер хранения, падение времени хранения УХН в ходе эксперимента и выход из строя изоляторов в процессе работы с высоким напряжением. Выполненные исследования позволяют дать некоторые практические рекомендации по преодолению этих трудностей.

1. „Звездчатость“ покрытий, получаемых магнетронным напылением.

Напыление металлического покрытия на прозрачное стекло хорошо иллюстрирует эту проблему. После напыления достаточно толстого (несколько сотен А) непрозрачного покрытия на стекло в свете яркой лампы на просвет хорошо видно большое количество мелких „отверстий“ с характерными размерами от долей до 
нескольких десятков $\mu \mathrm{m}$. Их наличие связано с остаточной пылью и остаточным загрязнением, практически всегда присутствующими на поверхности напыляемого образца и в вакуумной установке. Калибровочные измерения по пропусканию света показали, что в отдельных неблагоприятных случаях суммарная площадь „отверстий“ может достигать $0.1 \%$. При длительных временах хранения УХН это может приводить к заметным потерям нейтронов (в зависимости от граничной скорости материала подложки). Простое напыление добавочного слоя проблему не решает. Опыт показывает, что перед повторным напылением необходимо провести довольно жесткую чистку (промывку) поверхности с погружением в воду или спирт и с протиркой тканью. Относительная доля площади „отверстий“ после подобной обработки, как правило, возрастает вплоть до $\sim 1 \%$, но после проведения такой процедуры повторное напыление практически устраняет прозрачность покрытия: до 0.0001\% (контрольные измерения проводились по пропусканию света с калибровкой на заданное отверстие). При этом прочность напыленного слоя возрастает, и повторная промывка, если в ней возникает необходимость, уже не приводит к заметному увеличению суммарной площади „отверстий“'.

Напыление окиси бериллия на внутренние (рабочие) поверхности цилиндрических изоляторов осуществляется магнетронным распылением металлического бериллия в присутствии кислорода. Проверка изоляторов на высоковольтном стенде сразу после их напыления показывает значительный ток утечки. Очевидно, это связано с неполным окислением металла в процессе напылении. Поэтому окончательное окисление напыленного слоя производилось в воздушной атмосфере нагревом кольцевых изоляторов в закрытой печи до температуры $600^{\circ} \mathrm{C}$. Было установлено, что при толщине напыленного слоя более $3000 \AA$ время окисления отжигом весьма велико (несколько десятков $h$ ). При этом сохраняется риск неполного окисления, что при больших напряжениях может приводить к недопустимым по величине токам утечки и даже провоцировать пробои по поверхности изоляторов. Хорошие результаты были получены при следующей процедуре: после напыления первого слоя толщиной около $1500 \AA$ производилась промывка рабочей поверхности и отжиг в течение 10-12h при температуре $600^{\circ} \mathrm{C}$. Затем напылялся второй слой такой же толщины, и производился второй отжиг.

2. Падение времени хранения УХН в ходе длительного эксперимента из-за увеличения потерь нейтронов на рабочей поверхности камер хранения.

Как отмечалось выше, в процессе длительных непрерывных измерений ( $~ 50$ суток - цикл работы реактоpa) с коммутацией высокого напряжения наблюдалось плавное падение счета нейтронов после периода их хранения. Причиной этого является ухудшение качества рабочих поверхностей камер удержания УХН. После длительной работы с высоким напряжением на алюминиевых электродах можно было увидеть относительно крупные (порядка нескольких сантиметров) пятна потемнения первоначально однородной блестящей полированной поверхности с бериллиевым покрытием. Протиркой или промывкой эти потемнения не удаляются.

Из опыта работы специалистов нашего института с газовыми детекторами нам известно, что органические примеси (загрязнения поверхности или летучие соединения, остающиеся в вакууме) в процессе работы полимеризуются в газовом разряде и высаживаются прочным слоем на нитях детекторов. В нашей установке при „тренировке“ высокого напряжения (постепенном повышении напряжения с контролем токов утечки) происходят пробои в местах максимальной напряженности электрического поля, а именно на острых микродефектах полированных электродов. При пробоях острия распыляются и ликвидируются, напряжение поднимается. При этом распыляется не только бериллий на острие и вблизи него, но и алюминий острия. Алюминий обладает очень высокой химической активностью. Высаживаясь на поверхность, он затем сорбирует органические остаточные примеси в вакууме. В процессе тренировки высокого напряжения органика полимеризуется в разрядах и образует прочную пленку с большим содержанием водорода и большим сечением захвата нейтронов.

Микрообласти, оставшиеся в результате пробоев без бериллиевого покрытия, в лучшем случае, имеют граничную скорость отражения УХН, соответствующую алюминию $\left(v_{\text {critic }} \sim 3.2 \mathrm{~m} / \mathrm{s}\right)$, что при длительном хранении нейтронов эффективно обрезает верхнюю границу спектра УХН и уменьшает плотность хранящихся нейтронов.

Чтобы избежать распыления алюминия при разрядах и увеличить граничную скорость подложки, на которую напыляется бериллий, первоначально мы напыляем на электроды медь $\left(v_{\text {critic }} \sim 5.7 \mathrm{~m} / \mathrm{s}\right)$ толщиной $\sim 15 \mu \mathrm{m}$, а затем слоем толщиной около $1 \mu \mathrm{m}$, - бериллий. Изготовленные таким образом электроды перестали „загрязняться“ в процессе измерений, и время хранения УХН показало долговременную стабильность.

3. Выход из строя изоляторов в процессе тренировок и работы в режиме коммутации полярности высокого напряжения. Здесь под „выходом из строя“ мы понимаем такое изменение качества покрытия изоляторов, которое приводит к постепенному увеличению токов утечки до нескольких сотен $\mathrm{nA}$ и/или к увеличению числа поверхностных пробоев по изоляторам. И то, и другое требует снижения рабочего напряжения, как правило, до величин значительно ниже $100 \mathrm{kV}$, что существенно снижает чувствительность экспериментальной установки и делает набор полезной статистики в этих условиях очень малоэффективным. В таких случаях необходимо снимать испорченное покрытие изоляторов и проводить их новое напыление.

Анализ накопленных ранее экспериментальных наблюдений и исследования, выполненные на высоковольтном стенде, позволяют использовать полученный опыт и кратко сформулировать практические рекомендации, 
которые будут полезны при проведении аналогичных работ:

1) высоковольтную тренировку вновь напыленных электродов и изоляторов следует производить раздельно;

2) после напыления электродов по описанной выше процедуре следует собирать их с изоляторами без напыления; первичная тренировка электродов - процесс медленный, и для достижения рабочих напряжений около $\pm 250 \mathrm{kV}$ он может занимать один-два месяца (при некруглосуточной работе); в процессе тренировки при сильных пробоях по поверхности изолятора возможно ухудшение его качества: резкое и значительное увеличение тока утечки, требующее снижения высокого напряжения; в этом случае следует провести легкую механическую шлифовку поверхности изолятора и его промывку с последующим отжигом;

3) после завершения тренировки электродов следует напылить и отжечь изоляторы по описанной выше технологии; первичная тренировка изолятора с уже подготовленными электродами занимает 6-8h, за это время достигается напряжение $250 \mathrm{kV}$ на обеих полярностях; при некруглосуточной работе, после выключения источника высокого напряжения на $10-15 \mathrm{~h}$, подъем до прежнего достигнутого максимума занимает $2-3 \mathrm{~h}$.

Таким образом, на испытательном стенде была получена величина напряженности электрического поля в камерах хранения полномасштабной сборки ЭДМ-спектрометра около $27 \mathrm{kV} / \mathrm{cm}$. Ограничение определилось возможностями источника питания, поэтому предельное напряжение сборки пока не достигнуто. Токи утечки по изоляторам в конце тренировки составляли 3-5 nA, при этом пробоев и разрядов не было. До настоящего времени ни на каких действующих ЭДМ-установках с большими рабочими объемами такая напряженность электрического поля достигнута не была.

Подводя итог: описанная выше методика подготовки рабочих поверхностей высоковольтных камер удержания УХН обеспечивает необходимое качество и долговременную стабильность покрытий при работе с высоким напряжением. Полученная в рабочем объеме величина напряженности электрического поля $\sim 27 \mathrm{kV} / \mathrm{cm}$, при сохранении остальных параметров ЭДМ-спектрометра, практически вдвое увеличивает его чувствительность. При работе этой установки на существующем интенсивном пучке турбины УХН реактора ИЛЛ в Гренобле статистическая погрешность измерений величины ЭДМ за сутки будет составлять $\delta d_{n}<1 \cdot 10^{-25} e \mathrm{~cm}$, и такой чувствительности еще не имела ни одна экспериментальная установка для поиска ЭДМ-нейтрона. За 100 суток накопления данных в таких условиях может быть достигнута статистическая точность измерения величины ЭДМ-нейтрона лучше, чем $\delta d_{n}=1 \cdot 10^{-26} e \mathrm{~cm}$.

Авторы благодарят А.О. Полюшкина и И.А. Краснощекову за работу по модернизации высоковольтного источника и обеспечение автоматизации долговременных измерений.
Исследование выполнено в ФГБУ НИЦ „Курчатовский институт“ - ПИЯФ при поддержке гранта Российского научного фонда (проект № 14-22-00105-П).

\section{Список литературы}

[1] Backer C.A., Doyle D.D., Geltenbort P. et al. // Phys. Rev. Lett. 2006. Vol. 97. P. 131801-4.

[2] Serebrov A.P., Kolomenskiy E.A., Pirozhkov A.N. et al. // Phys. Rev. C. 2015. Vol. 92. P. 055501-15.

[3] Серебров А.П., Коломенский Э.А., Пирожкков А.Н. и др. // Кристаллография. 2016. Т. 61. № 1. С. 142-151.

[4] Алтарев И.С., Борисов Ю.В., Иванов С.Н. и др. // Препринт ПИЯФ. 2003. № 2514. С 1-41.

[5] Ласаков М.С., Полюшкин А.О., Серебров А.П. и др. // ЖТФ. 2016. Т. 86. Вып. 4. С. 125-129. 